\title{
The Sommerfeld Half-Plane Problem Revisited III: Parallel Plate Media with Mixed Boundary Conditions*)
}

\author{
A. E. Heins, Ann Arbor \\ Communicated by E. Meister
}

\begin{abstract}
A plane wave is normally incident upon an infinite stack of equally spaced parallel plates which are semi-infinite and not staggered. The plates satisfy the so-called "Rawlins" boundary conditions. This problem is formulated as a pair of simultaneous integral equations of the WienerHopf type and solved by a method proposed by A. E. Heins in 1950.
\end{abstract}

\section{Introduction}

We shall discuss here the reflection and transmission properties of a plane wave incident upon an infinite stack of unstaggered and equally spaced parallel plates. The plane wave will be taken at normal incidence to this structure. That is, its propagation normal will be both parallel to the plates and perpendicular to their edges. It will be assumed that each plate satisfies Rawlins' type of boundary conditions. That is, the total wave function vanishes on the lower side of each plate while its total normal derivative vanishes on the upper side. We will further assume that there is only specular reflection in the free space medium thereby giving us the possibility of two propagating modes in the parallel plate regions.

For the case of Dirichlet or Neumann type boundary conditions on each side of the plate, this problem was first solved by Carlson and Heins in 1944 [1]. An improved formulation and solution was given by Heins in 1956 [2]. The problems lead to scalar integral equations of the Wiener-Hopf type. The problem which we shall discuss here leads to a pair of such equations, and we shall show that the ideas we introduced in [3] will provide an effective solution of this system. Indeed, in the present instance, we shall be able to factor the matrix of the Fourier transforms of the kernels in much the same fashion that one can do it in the scalar case.

The problem we shall consider, has been solved by E. Lüneburg [5] by a generalization of the mode matching method. He states "the second case (the

*) The results of this paper were announced at the $X X^{\text {th }}$ General Assembly of the U.R.S.I. held in Washington, D.C. in August 1981. 
problem to be treated here) is not solvable by these methods (the method of Wiener and Hopf) but yields to the new method" (a generalization of the mode matching method).

\section{The Formulation of the Problem}

Let us recall that if a plane wave is normally incident upon an infinite stack of semi-infinite, equally spaced unstaggered plates, (see Fig. 1).

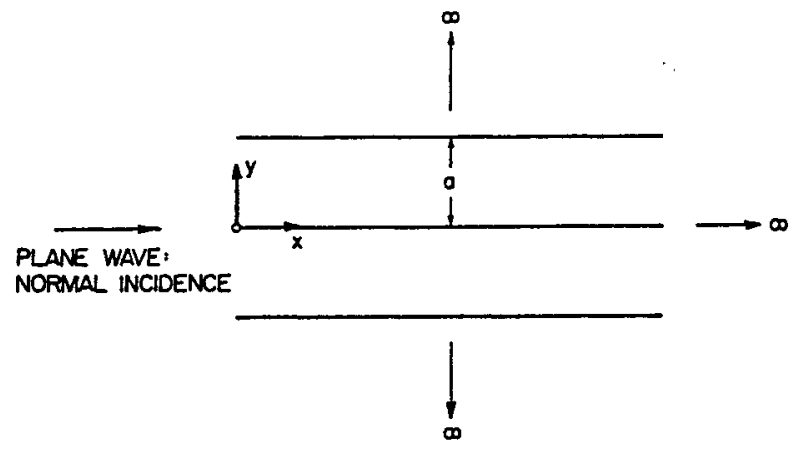

Fig. 1

$$
\begin{aligned}
& \phi(x, y)=\exp (\mathrm{i} k x)+R \exp (-\mathrm{i} k x) \\
& -\int_{0}^{\infty}\left[G\left(x, y ; x^{\prime}, 0\right) \phi_{y^{\prime}}\left(x^{\prime},+0\right)-\phi\left(x^{\prime},+0\right) \frac{\partial G}{\partial y^{\prime}}\left(x, y ; x^{\prime},+0\right)\right] \mathrm{d} x^{\prime} \\
& +\int_{0}^{\infty}\left[G\left(x, y, x^{\prime}, a-\right) \phi_{y^{\prime}}\left(x^{\prime}, a-\right)-\phi\left(x^{\prime}, a-\right) \frac{\partial G}{\partial y^{\prime}}\left(x, y ; x^{\prime}, a-\right)\right] \mathrm{d} x^{\prime}
\end{aligned}
$$

is a representation of the solution of the equation

$$
\phi_{x x}+\phi_{y y}+k^{2} \phi=0
$$

which incorporates the Sommerfeld radiation condition for plane waves, the incident wave $\exp (\mathrm{i} k x)$ and the periodic nature of the structure. The Green's function $G\left(x, y ; x^{\prime}, y^{\prime}\right)$ is a solution of the equation

$$
G_{x x}+G_{y y}+k^{2} G=0
$$

save at the point $P(x, y)=P^{\prime}\left(x^{\prime}, y^{\prime}\right)$ where it has a logarithmic singularity. It also satisfies the periodic boundary conditions

$$
G\left(x,-\alpha ; x^{\prime}, y^{\prime}\right)=G\left(x, a-\alpha ; x^{\prime}, y^{\prime}\right)
$$

and $\left.\quad G_{y}\left(x, y ; x^{\prime}, y^{\prime}\right)\right|_{y=-\alpha}=\left.G_{y}\left(x, y ; x^{\prime}, y\right)\right|_{y=\alpha-\alpha}$

where $\alpha$ is a real parameter. Such a Green's function was constructed by the author in 1957 [2] and it has the Fourier integral representation 


$$
\begin{aligned}
G\left(x, y ; x^{\prime}, y^{\prime}\right)= & \frac{1}{2 \pi} \int_{\mathscr{Z}} \frac{\left[\sin \sigma\left(y^{\prime}-y\right)-\sin \sigma\left(y^{\prime}-y+a\right)\right]}{2 \sigma(1-\cos \sigma a)} \\
& \cdot[\operatorname{expiw}(x-x)] \mathrm{d} w, \quad y>y^{\prime} \\
= & \frac{1}{2 \pi} \int_{\mathscr{L}} \frac{\left[\sin \sigma\left(y^{\prime}-y-a\right)-\sin \sigma\left(y^{\prime}-y\right)\right]}{2 \sigma(1-\cos \sigma a)} \\
& \cdot\left[\operatorname{expi} w\left(x-x^{\prime}\right)\right] \mathrm{d} w, \quad y<y^{\prime} .
\end{aligned}
$$

Here $\sigma=\sqrt{k^{2}-w^{2}}$ and is real and positive for $w=0 . \mathscr{L}$ is a path of integration parallel to the real axis of the $w$ plane and it is drawn in the strip $-\sqrt{\frac{4 \pi^{2}}{a^{2}}-k^{2}}$
$<\operatorname{Im} w<0$. (2.4) may be simplified to

$$
\begin{aligned}
G\left(x, y ; x^{\prime}, y\right) & =-\frac{1}{2 \pi} \int_{\mathscr{Y}} \frac{\cos \sigma\left(y^{\prime}-y+\frac{a}{2}\right)}{2 \sigma \sin \frac{\sigma a}{2}}\left[\operatorname{expiw}\left(x-x^{\prime}\right)\right] \mathrm{d} w, \quad y>y^{\prime} \\
& =-\frac{1}{2 \pi} \int_{\mathscr{L}} \frac{\cos \sigma\left(y^{\prime}-y-\frac{a}{2}\right)}{2 \sigma \sin \frac{\sigma a}{2}}\left[\operatorname{expiw}\left(x-x^{\prime}\right)\right] \mathrm{d} w, \quad y<y^{\prime}
\end{aligned}
$$

and

From (2.4a) we may conclude that

and

$$
\begin{aligned}
& G\left(x, y ; x^{\prime}, y^{\prime}\right)=-\frac{\sin k\left(x-x^{\prime}\right)}{a k}, \quad x-x^{\prime} \rightarrow \infty \\
&=\frac{\cos \frac{2 \pi}{a}\left(y^{\prime}-y\right)}{\sqrt{4 \pi^{2}-a^{2} k^{2}}}\left[\exp \sqrt{\frac{4 \pi^{2}}{a^{2}}-k^{2}}\left(x-x^{\prime}\right)\right] ; \\
& x-x^{\prime} \rightarrow-\infty .
\end{aligned}
$$

Here we propose to invoke "Rawlins" [6] type boundary conditions, rather than those used by Carlson and Heins [1], so that for $x>0$, we obtain the system of integral equations of the Wiener-Hopf type

$$
\begin{aligned}
0= & \exp (i k x)+R \exp (-i k x) \\
& -\int_{0}^{\infty} G\left(x, 0 ; x^{\prime}, 0\right) \phi_{y^{\prime}}\left(x^{\prime},+0\right) \mathrm{d} x^{\prime}-\frac{\phi(x, a-)}{2}
\end{aligned}
$$

and

(2.5b) $0=\frac{-\phi_{y}(x,+0)}{2}-\frac{\partial^{2}}{\partial y \partial y^{\prime}} \int_{0}^{\infty} G\left(x, y ; x^{\prime}, y\right) \phi\left(x^{\prime}, y\right) \mathrm{d} x^{\prime}, \quad y=y^{\prime}=a-$.

By Rawlin's type boundary conditions we mean $\phi(x,+0)=\frac{\partial \phi}{\partial y}(x, a-)=0$,
$x>0$. 
Following Wiener and Hopf, we rewrite this system, valid for $-\infty<x$ $<\infty$ as follows. We put

$$
\begin{aligned}
f_{1}(x) & \equiv \phi_{y}(x,+0), & & x>0 \\
& =0, & & x<0 \\
f_{2}(x) & =\phi(x, a-), & & x>0 \\
& =0, & & x<0 \\
F_{1}(x) & =\phi_{y}(x, a-), & & x<0 \\
& =0, & & x>0 \\
F_{2}(x) & \equiv \phi(x,+0), & & x<0 \\
& =0, & & x<0 \\
\phi^{*}(x) & \exp (\mathrm{i} k x)+R \exp (-\mathrm{i} k x), & & x>0 \\
& \equiv 0, & & x<0 .
\end{aligned}
$$

Hence the system now may be written as

(2.6a) $F_{1}(x)=-\frac{f_{1}(x)}{2}-\frac{\partial^{2}}{\partial y \partial y^{\prime}} \int_{-\infty}^{\infty} G\left(x, y ; x^{\prime}, y^{\prime}\right) f_{2}\left(x^{\prime}\right) \mathrm{d} x^{\prime}, \quad y=y^{\prime}=a-$

and

(2.6b) $F_{2}(x)=\phi^{*}(x)-\frac{f_{2}(x)}{2}-\int_{-\infty}^{\infty} G\left(x,+0 ; x^{\prime},+0\right) f_{1}\left(x^{\prime}\right) \mathrm{d} x^{\prime}$.

A final comment is required regarding the modes of propagation which we permit. Specular reflection requires that $0<a k<2 \pi$. On the other hand, the lowest mode which can propagate in the parallel plate region requires $\pi / 2$ $<a k<3 \pi / 2$. Hence if only specular reflection is permitted, we may have one or two modes propagating in the parallel plate region. The propagation constant for one mode propagating is $\left(k^{2}-\frac{\pi^{2}}{4 a^{2}}\right)^{1 / 2}>0$, while for two modes, we have the constants $\left(k^{2}-\frac{\pi^{2}}{4 a^{2}}\right)^{1 / 2}>0$ and $\left(k^{2}-\frac{9 \pi^{2}}{4 a^{2}}\right)^{1 / 2}>0$. We shall only treat the case $\pi / 2<a k<3 \pi / 2$ since we will not add anything new to our method by permitting $a k$ to be greater than $3 \pi / 2$.

\section{The Fourier Transform of the System (2.6)}

The determination of the Fourier transform of the system (2.6) is straight-forward. We know, for example, that 


$$
F_{1}(x)=F_{2}(x)=O\left(\exp x \sqrt{\frac{4 \pi^{2}}{a^{2}}-k^{2}}\right), x \rightarrow-\infty,
$$

as we can conclude directly from the system (2.6). We also assume for the present, and shall verify, that $F_{1}(x), F_{2}(x), f_{1}(x)$ and $f_{2}(x)$ are integrable in the neighborhood of the origin. Since the parallel plate region can only sustain one or two propagating modes, we have that $f_{1}(x)$ and $f_{2}(x)=O(1), x \rightarrow \infty$. Hence the Fourier transforms of $F_{1}(x)$ and $F_{2}(x)$, that is,

$$
\int_{-\infty}^{0} \exp (-\mathrm{i} w x) F_{j}(x) \mathrm{d} x=\hat{F}_{j}(w), \quad j=1,2
$$

are analytic in the upper half-plane $\operatorname{Im} w>-\sqrt{\frac{4 \pi^{2}}{a^{2}}-k^{2}}$. Furthermore, the Fourier transforms of $f_{1}(x)$ and $f_{2}(x)$, that is

$$
\int_{0}^{\infty} \exp (-\mathrm{i} w x) f_{j}(x) \mathrm{d} x=f_{j}(w), \quad j=1,2
$$

are analytic in the lower half-plane $\operatorname{Im} w<0$.

We also require the bilateral Fourier transform of $G(x, 0 ; 0,0)$ and $G_{y y^{\prime}}(x, y ; 0, y)$ for $y=y^{\prime}=a$. Here we have

$$
\int_{-\infty}^{\infty} \exp (-\mathrm{i} w x) G(x, 0 ; 0,0) \mathrm{d} x=\frac{-\sin a \sqrt{k^{2}-w^{2}}}{2 \sqrt{k^{2}-w^{2}}\left[1-\cos a \sqrt{k^{2}-w^{2}}\right]}
$$

and

$$
\begin{aligned}
& \frac{\partial^{2}}{\partial y \partial y^{\prime}} \int_{-\infty}^{\infty} \exp (-\mathrm{i} w x) G(x, y ; 0, y) \mid \mathrm{d} x \\
& =-\frac{\sqrt{k^{2}-w^{2}} \sin a \sqrt{k^{2}-w^{2}}}{2\left[1-\cos a \sqrt{k^{2}-w^{2}}\right]}
\end{aligned}
$$

(See [2] for the derivation of these transforms.) In virtue of the behavior of $G\left(x, y^{\prime} ; 0, y\right)$ when $|x| \rightarrow \infty,(3.1)$ and (3.2) are analytic in the strip $-\sqrt{\frac{4 \pi^{2}}{a^{2}}-k^{2}}$ $<\operatorname{Im} w<0$. The transform of $\phi^{*}(x)$ is clearly analytic in the lower half-plane $\operatorname{Im} w<0$. Hence $\hat{F}_{j}(w), \hat{f}_{j}(w)(j=1,2), \hat{\phi}^{*}(w)$ and the transform of $G\left(x, y ; 0, y^{\prime}\right)$ are all analytic in a common strip $-\sqrt{\frac{9 \pi^{2}}{4 a^{2}}-k^{2}}<\operatorname{Im} w<0$. It is therefore permissable to apply the Fourier transform to equations (2.6a) and (2.6b) to obtain

$$
F_{2}(w)=\frac{1}{\mathrm{i}}\left[\frac{1}{w-k}+\frac{R}{w+k}\right]+\frac{\cot \left[a \sqrt{k^{2}-w^{2}} / 2\right]}{2 \sqrt{k^{2}-w^{2}}} \hat{f}_{1}(w)-\frac{\hat{f}_{2}(w)}{2}
$$


and $f_{1}(w)=-\frac{\hat{f}_{1}(w)}{2}+\frac{\sqrt{k^{2}-w^{2}} \cot \left[a \sqrt{k^{2}-w^{2}} / 2\right] f_{2}(w)}{2}$.

In matrix notation we have

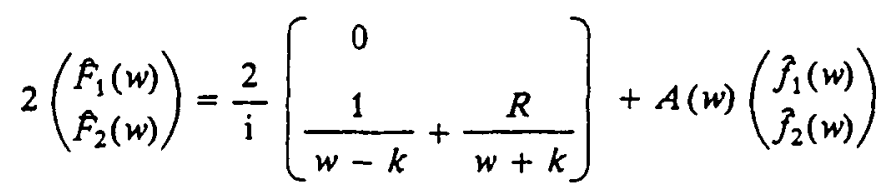

where $A(w)=\left[\begin{array}{cc}-1 & \sqrt{k^{2}-w^{2}} \cot \left(a \sqrt{k^{2}-w^{2}} / 2\right) \\ \frac{\cot \left(a \sqrt{k^{2}-w^{2}} / 2\right)}{\sqrt{k^{2}-w^{2}}} & -1\end{array}\right]$.

Observe that $\operatorname{det} A(w)=-\cos a \sigma / \sin ^{2} a \sigma / 2$ and although it has no poles in the strip $-\sqrt{\frac{4 \pi^{2}}{a^{2}}-k^{2}}<\operatorname{Im} w<0$, it does have a zero at $\operatorname{Im} w=-\sqrt{\frac{9 \pi^{2}}{4 a^{2}}-k^{2}}$.

(Recall that we have assumed that only the lowest mode propagates in the duct.) Hence, as we shall see presently, it is necessary that we take the smaller strip $-\sqrt{\frac{9 \pi^{2}}{4 a^{2}}-k^{2}}<\operatorname{Im} w<0$ as the strip of analyticity.

\section{The Factoring of the Matrix $A(w)$}

If we could write the matrix $A(w)=A_{-}(w) A_{+}(w)=A_{+}(w) A_{-}$(w) such that all elements in $A_{-}(w)$ would be analytic in the lower half-plane Im $w$ $<0$, and $\operatorname{det} A_{-}(w)$ would be free of zeros and poles there, while $A_{+}(w)$ would have similar properties in the upper half-plane $\operatorname{Im} w>-\sqrt{\frac{9 \pi^{2}}{4 a^{2}}-k^{2}}$, we would be in a position to repeat the original argument of Wiener and Hopf. The need for this last inequality will be given subsequently. That it is possible to repeat this argument here [3] and in several cases which we shall discuss at a later date, is primarily due to the fact that we are dealing with $2 \times 2$ matrices and we can overcome the algebraic problems which are encountered in these cases. Note the position of the factor $\sigma(w)$ in the matrix $B(w)$ which we shall now introduce. We write

$$
A(w)=\exp [B(w)]
$$

and we obtain by Sylvester's theorem [4]

$$
B(w)=\frac{\left(\begin{array}{cc}
1 & \sigma \\
1 / \sigma & 1
\end{array}\right)}{2} \ln \left(-1+\cot \frac{a \sigma}{2}\right)+\frac{\left(\begin{array}{cc}
1 & -\sigma \\
-1 / \sigma & 1
\end{array}\right)}{2} \ln \left(-1-\cot \frac{a \sigma}{2}\right) .
$$


We can now rearrange the entries in the matrix $B(w)$ as follows.

$$
B(w)=I \ln D(w)+C(w) \ln E(w) .
$$

Here

$$
C(w)=\left(\begin{array}{ll}
0 & \sigma \\
\frac{1}{\sigma} & 0
\end{array}\right)
$$

$C^{2}(w)=I$ and $I$ is the identity matrix. We also have

$$
D(w)=\sqrt{1-\cot ^{2} \frac{a \sigma}{2}} \text { and } E(w)=\sqrt{\frac{1-\cot \frac{a \sigma}{2}}{1+\cot \frac{a \sigma}{2}}} .
$$

This rearrangement of $B(w)$ is permissible since $I$ and $C(w)$ obviously commute.

We shall for the present, assume that we can derive an additive decomposition of the matrix $B(w)$ such that the elements of $B_{+}(w)$, that is,

$$
B_{+}(w)=I \ln D_{+}(w)+C(w) \ln H_{1}(w)
$$

and their determinant are analytic in the upper half-plane $\operatorname{Im} w>-\sqrt{\frac{9 \pi^{2}}{4 \pi^{2}}-k^{2}}$.
Similarly these elements of $B_{-}(w)$ are given by

$$
B_{-}(w)=I \ln D_{-}(w)+C(w) \ln H_{2}(w)
$$

and are analytic in the lower half-plane $\operatorname{Im} w<0$ and also have a non-vanishing determinant there. We shall demonstrate in the Appendix that the elements of the matrix $C(w)$ weighted with $\ln E(w)$ may be written as

$$
\left(\frac{\ln E(w)}{\sigma}\right)_{+}+\left(\frac{\ln E(w)}{\sigma}\right)_{-}=\frac{\ln H_{1}(w)}{\sigma}+\frac{\ln H_{2}(w)}{\sigma}
$$

where the subscript + or - denotes analyticity in the upper or lower half-planes which we have already described. Furthermore, as we shall see in the Appendix,

$$
\left(\frac{\ln E(w)}{\sigma}\right)_{+}=\frac{\ln H_{1}(w)}{\sigma} \text { and }\left(\frac{\ln E(w)}{\sigma}\right)_{-}=\frac{\ln H_{2}(w)}{\sigma} \text {. }
$$

It is this decomposition which enables us to proceed with the present problem. There is, at present, no guarantee that we can find decomposition of the form (4.1) and (4.1 a) in general. For the case at hand, we have

$$
A_{+}(w)=\exp \left[B_{+}(w)\right]=\frac{D_{+}(w)}{2}\left\{\left(H_{1}+\frac{1}{H_{1}}\right) I+\left(H_{1}-\frac{1}{H_{1}}\right) C(w)\right\} .
$$

In component form we have 


$$
A_{+}(w)=\frac{D_{+}(w)}{2}\left[\begin{array}{cc}
H_{1}+\frac{1}{H_{1}} & \sigma\left(H_{1}-\frac{1}{H_{1}}\right) \\
\frac{H_{1}-\frac{1}{H_{1}}}{\sigma} & H_{1}+\frac{1}{H_{1}}
\end{array}\right]
$$

and it is clear that the determinant of this matrix does not vanish. The expressions for $D_{+}(w), D_{-}(w), H_{1}(w)$ and $H_{2}(w)$ will be derived in the Appendix. It is easy to show that $A_{+}(w)$ and $A_{-}(w)$ commute and $A_{+}(w) A_{-}(w)=A(w)$.

We now proceed to the Wiener-Hopf separation. The vector equation (3.3) may be written as

$$
A_{+}(w) A_{-}(w)\left(\begin{array}{l}
f_{1}(w) \\
f_{2}(w)
\end{array}\right)-2 \mathrm{i}\left[\begin{array}{c}
0 \\
\frac{1}{(w-k)}+\frac{R}{w+k}
\end{array}\right)=2\left(\begin{array}{l}
\hat{F}_{1}(w) \\
F_{2}(w)
\end{array}\right)
$$

or

$$
A_{-}(w)\left(\begin{array}{l}
\hat{f}_{1}(w) \\
\hat{f}_{2}(w)
\end{array}\right)-2 \mathrm{i} A_{+}^{-1}(w)\left(\begin{array}{c}
0 \\
\frac{1}{(w-k)}+\frac{R}{w+k}
\end{array}\right)=2 A_{+}^{-1}(w)\left(\begin{array}{l}
f_{1}(w) \\
F_{2}(w)
\end{array}\right)
$$

where

$$
\begin{aligned}
& A_{+}^{-1}(w) \\
& =\frac{1}{2 D_{+}(w)}\left(\begin{array}{cc}
H_{1}(w)+1 / H_{1}(w) & -\sigma(w)\left[H_{1}(w)-1 / H_{1}(w)\right] \\
-\left[H_{1}(w)-1 / H_{1}(w)\right] / \sigma(w) & H_{1}(w)+1 / H_{1}(w)
\end{array}\right) .
\end{aligned}
$$

Hence equation (4.2) reduces to

$$
\begin{aligned}
& A_{-}(w)\left(\begin{array}{l}
\hat{f}_{1}(w) \\
\hat{f}_{2}(w)
\end{array}\right) \\
& -\frac{\mathrm{i}}{D_{+}(w)}\left(\frac{1}{w-k}+\frac{R}{w+k}\right)\left(\begin{array}{c}
-\sigma(w)\left[H_{1}(w)-1 / H_{1}(w)\right] \\
H_{1}(w)+1 / H_{1}(w)
\end{array}\right) \\
& =2 A_{+}^{-1}(w)\left(\begin{array}{l}
\hat{F}_{1}(w) \\
\hat{F}_{2}(w)
\end{array}\right)
\end{aligned}
$$

and is analytic in the strip $-\sqrt{\frac{9 \pi^{2}}{4 \pi a^{2}}-k^{2}}<\operatorname{Im} w<0$. Now we employ the same device which is available for the scalar case (see for example [1]), and obtain

$$
\begin{aligned}
A_{-}(w)\left(\begin{array}{l}
f_{1}(w) \\
f_{2}(w)
\end{array}\right) & -\frac{\mathrm{i}}{D_{+}(k)(w-k)}\left(\begin{array}{c}
0 \\
H_{1}(k)+1 / H_{1}(k)
\end{array}\right) \\
& -\frac{\mathrm{i} R}{D_{+}(-k)(w+k)}\left(\begin{array}{c}
0 \\
H_{1}(-k)+1 / H_{1}(-k)
\end{array}\right)
\end{aligned}
$$




$$
\begin{gathered}
=2 A_{+}^{-1}(w)\left(\begin{array}{l}
\hat{F}_{1}(w) \\
F_{2}(w)
\end{array}\right)-\frac{\mathrm{i}}{(w-k)}\left[\begin{array}{l}
\sigma(w)\left[H_{1}(w)-1 / H_{1}(w)\right] / D_{+}(w) \\
\left\{\left[H_{1}(k)+1 / H_{1}(k)\right] / D_{+}(k)\right. \\
\left.-\left[H_{1}(w)+1 / H_{1}(w)\right] / D_{+}(w)\right\}
\end{array}\right) \\
-\frac{\mathrm{i} R}{(w+k)}\left(\begin{array}{l}
\sigma(w)\left[H_{1}(w)-1 / H_{1}(w)\right] / D_{+}(w) \\
\left\{\left[H_{1}(-k)+1 / H_{1}(-k)\right] / D_{+}(-k)\right. \\
\left.-\left[H_{1}(w)+1 / H_{1}(w)\right] / D_{+}(w)\right\}
\end{array}\right]
\end{gathered}
$$

The left side of equation (4.4) is analytic in the lower half-plane $\operatorname{Im} w<0$, while the right side is analytic in the upper half-plane $\operatorname{Im} w>-\sqrt{\frac{9 \pi^{2}}{4 a^{2}}-k^{2}}$ and both sides are analytic in a common strip of the $w$ plane. Hence each side is analytic everywhere and is equal to a vector whose components are entire functions. That is, for $\operatorname{Im} w<0$ we have

$$
\begin{aligned}
& A_{-}(w)\left(\begin{array}{l}
\hat{f}_{1}(w) \\
f_{2}(w)
\end{array}\right)-\frac{\mathrm{i}}{D_{+}(k)(w-k)}\left(\begin{array}{c}
0 \\
H_{1}(k)+1 / H_{1}(k)
\end{array}\right) \\
& -\frac{\mathrm{i} R}{(w+k) D_{+}(-k)}\left(\begin{array}{c}
0 \\
H_{1}(-k)+1 / H_{1}(-k)
\end{array}\right)=\left(\begin{array}{l}
E_{1}(w) \\
E_{2}(w)
\end{array}\right)
\end{aligned}
$$

where $E_{1}(w)$ and $E_{2}(w)$ are entire functions yet to be determined. Note that since $A_{-}^{-1}(w)$ is analytic in the lower half-plane $\operatorname{Im} w<0$, that is, its elements are and further $\operatorname{det} A_{-}(w) \neq 0$ in this half-plane, we can solve for the vector $\left(\hat{f}_{1}(w)\right.$, $\left.f_{2}(w)\right)$. Similar remarks are available for $\left(\hat{F}_{1}(w), \hat{F}_{2}(w)\right)$.

\section{The Determination of the Entire Functions $E_{1}(w)$ and $E_{2}(w)$}

We are now in a position to determine the entire functions $E_{1}(w)$ and $E_{2}(w)$. As in the scalar case, we shall find that the Liouville theorem will produce them up to an arbitrary constant if we invoke the edge conditions for $f_{1}(x), f_{2}(x)$, $F_{1}(x)$ and $F_{2}(x)$. It will then be necessary to verify that the Riemann-Lebesgue lemma is satisfied for $\hat{f}_{1}(w), \pi_{2}(w), \hat{F}_{1}(w)$ and $\hat{F}_{2}(w)$ in the appropriate half-plane and this will produce the evaluation of the constant.

Let us recall the properties we seek for $\hat{f}_{1}(w), \hat{f}_{2}(w), \hat{F}_{1}(w)$ and $\hat{F}_{2}(w)$. We need the $\vec{f}_{1}(w)$ and $\hat{f}_{2}(w)$ which are analytic in the lower half-plane $\operatorname{Im} w<0$ and the $\hat{F}_{1}(w)$ and $\hat{F}_{2}(w)$ which are analytic in the upper half-plane $\operatorname{Im} w$ $>-\sqrt{\frac{9 \pi^{2}}{4 a^{2}}-k^{2}}$. In virtue of the fact that $f_{1}(x)=O\left(x^{-3 / 4}\right), x \rightarrow 0^{+}$we have by the Abelian theorem for the unilateral Fourier transform that $f_{1}(w)=$ $O\left(w^{-1 / 4}\right), \operatorname{Im} w<0,|w| \rightarrow \infty$. Similarly since $f_{2}(x)=O\left(x^{1 / 4}\right), x \rightarrow 0^{+}, f_{2}(w)=$ $O\left(w^{-5 / 4}\right)|w| \rightarrow \infty$ in the half-plane $\operatorname{Im} w<0$. In a like manner, $\hat{F}_{1}(w)=$ $O\left(w^{-1 / 4}\right)$ and $f_{2}(w)=O\left(w^{-5 / 4}\right)$ when $\operatorname{Im} w>-\sqrt{\frac{9 \pi^{2}}{4 a^{2}}-k^{2}},|w| \rightarrow \infty$. 
We shall show in the Appendix that $H_{1}(w)=O\left(w^{-1 / 4}\right),|w| \rightarrow \infty$, $\operatorname{Im} w>-\sqrt{\frac{9 \pi^{2}}{4 a^{2}}-k^{2}}$ while $H_{2}(w)=O\left(w^{1 / 4}\right),|w| \rightarrow \infty, \operatorname{Im} w<0$. Furthermore $D_{+}(w)=O(1),|w| \rightarrow \infty, \operatorname{Im} w>-\sqrt{\frac{9 \pi^{2}}{4 a^{2}}-k^{2}}$ and $D_{-}(w)=O(1)$, $|w| \rightarrow \infty$, Im $w<0$. This implies $E_{1}(w)=O(1)$ and $E_{2}(w)=0, \operatorname{Im} w<0$, $|w| \rightarrow \infty$. In the upper half-plane we have that $E_{1}(w)=O\left(w^{1 / 4}\right)$ and $E_{2}(w)=0$ when $|w| \rightarrow \infty$. Now the Liouville theorem tells us that $E_{2}(w)=0$. As for $E_{1}(w)$, it is clear that since it is an entire function it can only be $O(1)$ and therefore the Liouville theorem tells us that it is an arbitrary constant which we label $a_{0}$.

Now we are in a position to determine $\vec{f}_{1}(w)$ and $\vec{f}_{2}(w)$ after we verify that Riemann-Lebesgue lemma is satisfied. From (4.5) we have

$$
\begin{gathered}
\left(\begin{array}{l}
f_{1}(w) \\
f_{2}(w)
\end{array}\right)-\frac{\mathrm{i}\left[H_{1}(k)+1 / H_{1}(k)\right]}{2 D_{+}(k) D_{-}(w)(w-k)}\left(\begin{array}{c}
-\sigma\left[H_{2}(w)-1 / H_{2}(w)\right] \\
H_{2}(w)+1 / H_{2}(w)
\end{array}\right) \\
-\frac{\mathrm{i} R\left[H_{1}(-k)+1 / H_{1}(-k)\right]}{2 D_{+}(-k) D_{-}(w)(w+k)}\left(\begin{array}{c}
-\sigma\left[H_{2}(w)-1 / H_{2}(w)\right] \\
H_{2}(w)+1 / H_{2}(w)
\end{array}\right) \\
=\frac{a_{0}}{2 D_{-}(w)}\left[\begin{array}{c}
H_{2}(w)+1 / H_{2}(w) \\
-\frac{H_{2}(w)-1 / H_{2}(w)}{\sigma}
\end{array}\right] .
\end{gathered}
$$

Clearly $\hat{f}_{2}(w)$ obeys the lemma but $\hat{f}_{1}(w)$ does not. However, if we choose

$$
a_{0}=-\frac{\mathrm{i}\left[H_{1}(k)+1 / H_{1}(k)\right]}{D_{+}(k)}-\frac{\mathrm{i} R\left[H_{1}(-k)+1 / H_{1}(-k)\right]}{D_{+}(-k)}
$$

then $f_{1}(w)$ will also obey the lemma. Indeed we will now have, as we expected, that $\hat{f}_{1}(w)=O\left(w^{-1 / 4}\right)$ and $f_{2}(w)=O\left(w^{-5 / 4}\right),|w| \rightarrow \infty, \operatorname{Im} w<0$. The same constant $a_{0}$ takes care of the equations involving $F_{1}(w)$ and $F_{2}(w)$.

\section{The Determination of the Reflection and Transmission Amplitudes}

With the determination of $\hat{f}_{2}(w)$ we are now in a position to determine the amplitude of the reflected wave in free space as well as that of the transmitted wave in the duct. If we examine the functional form of $f_{2}(w)$, we observe that it has two real simple poles at $w= \pm \varkappa$, all other poles are simple and imaginary and lie in the upper half-plane $\operatorname{Im} w>0$. Indeed as we shall show in the Appendix, it is a single valued analytic function in the $w$ plane save for these poles. This fact can be accounted for on physical grounds.

We turn then to the examination of the $\lim _{x \rightarrow \infty} f_{2}(x)$ in order to determine the required amplitudes. What is required then is the residue of $f_{2}(w)$ at $w= \pm \varkappa$. On the other hand, $H_{2}(w) / D_{-}(w)$ has simple poles at $w= \pm \varkappa$, while 
$D_{-}(w) / H_{2}(w)$ does not have poles at $w= \pm x$. We therefore obtain these residues from the term

$$
\frac{\mathrm{i} H_{2}(w)}{D_{-}(w)}\left[\frac{H_{1}(k)+1 / H_{1}(k)}{D_{+}(k)(w-k)}+\frac{R\left[H_{1}(-k)+1 / H_{1}(-k)\right]}{D_{+}(-k)(w+k)}\right]
$$

the remaining poles supplying those terms which vanish when $x \rightarrow \infty$. There is an immediate simplification since $H_{1}(k)+1 / H_{1}(k)=H_{1}(-k)+1 / H_{1}(-k)=2$ (see A.10). Furthermore by putting in the explicit expressions for $\mathrm{H}_{2}(w)$ and $D_{-}(w),(6.1)$ becomes

$$
\begin{aligned}
& \frac{\mathrm{i}}{2}\left[\frac{1}{D_{+}(k)(w-k)}+\frac{R}{D_{+}(-k)(w+k)}\right] \frac{\pi \sigma(w)[2 a \sigma(w)+\pi] \exp [\chi(w) / 2]}{8 a\left(\chi^{2}-w^{2}\right)} \\
& \cdot \prod_{n=1}^{\infty}\left\{\left[\frac{1+(2 a \sigma(w)+\pi) / 4 n \pi)}{1+(2 a \sigma(w)-\pi) / 4 n \pi)}\right] \exp (-1 / 2 n)\right\} M^{1 / 2}(w) L^{1 / 2}(w)
\end{aligned}
$$

$$
\prod_{n=1}^{\infty}\left[\sqrt{1-a^{2} k^{2} / 4 n^{2} \pi^{2}}+\mathrm{i} a w / 2 n \pi\right] \exp (-\mathrm{i} a w / 2 n \pi]
$$

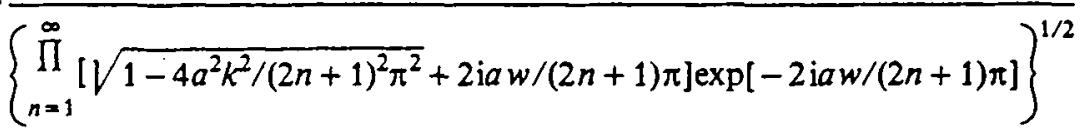

The functions $L(w)$ and $M(w)$ have been derived in the Appendix and are

$$
L(w)=\prod_{n=1}^{\infty} \frac{\sqrt{(1-1 / 4 n)^{2}-a^{2} k^{2} / 4 n^{2} \pi^{2}}+\mathrm{i} a w / 2 n \pi}{\sqrt{(1+1 / 4 n)^{2}-a^{2} k^{2} / 4 n^{2} \pi^{2}}+\mathrm{i} a w / 2 n \pi} \exp (1 / 2 n)
$$

and $M(w)=$

$$
\prod_{n=1}^{\infty} \frac{(1-1 / 4 n) \sigma(w)+a k^{2} / 2 n \pi+\mathrm{i} w \sqrt{(1-1 / 4 n)^{2}-a^{2} k^{2} / 4 n^{2} \pi^{2}}}{(1+1 / 4 n) \sigma(w)+a k^{2} / 2 n \pi+\mathrm{i} w \sqrt{(1+1 / 4 n)^{2}-a^{2} k^{2} / 4 n^{2} \pi^{2}}} \exp (1 / 2 n) .
$$

From (6.2) we can, by calculating the residues at the poles $w= \pm \varkappa$, find the limit $f_{2}(x), x \rightarrow \infty$. However, the residue at $w=-\varkappa$ implies a wave from the far right in the duct which we have excluded. Therefore the coefficient of this duct term vanishes and gives us $R$ immediately. That is,

$$
-\frac{1}{D_{+}(k)(k+x)}+\frac{R}{D_{+}(-k)(k-x)}=0
$$

or

$$
R=\frac{D_{+}(-k)(k-x)}{D_{+}(k)(k+x)} \quad \pi / 2<a k<3 \pi / 2
$$

Since $D_{+}(k)$ is conjugate to $D_{+}(-k)$, we have finally that 


$$
|R|=\frac{k-x}{k+x} .
$$

Now it is possible to calculate $|T|$, the amplitude of the duct wave travelling to the right, by a conservation condition [2]. This condition is

$$
k\left[1-|R|^{2}\right]=\varkappa|T|^{2} / 2
$$

from which we obtain

$$
|T|^{2}=\frac{8 k^{2}}{(k+x)^{2}} \quad \pi / 2<a k<3 \pi / 2 .
$$

However (6.2) and (6.3) of fer an independent verification of (6.4) which we shall now carry out.

If we now calculate the residue of (6.2) at $w=\varkappa$, eliminate $R$ by (6.3), observe that $\left|D_{+}(k)\right|=\left|D_{+}(-k)\right|=1$, and then take the absolute value of (6.2), we obtain

$$
\begin{gathered}
\frac{k \pi}{2(k+x)} \prod_{n=1}^{\infty}\left(1+\frac{1}{2 n}\right) e^{-1 / 2 n}\left[\prod_{n=1}^{\infty} \frac{\mathrm{e}^{1 / 2 n}}{1+1 / 2 n}\right]^{1 / 2} \\
\cdot\left[\prod_{n=1}^{\infty} \frac{1-1 / 2 n}{1+1 / 2 n} \mathrm{e}^{+1 / n}\right]^{1 / 4}\left[\prod_{n=1}^{\infty}\left(1-\frac{1}{16 n^{2}}\right)\right]^{1 / 2} \\
\cdot\left[\prod_{n=1}^{\infty}\left(1-\frac{1}{(2 n+1)^{2}}\right)\right]^{-1 / 4} .
\end{gathered}
$$

The product factors appear in the same order as they do in (6.2). Now we can combine the first three factors of (6.5) since they are absolutely convergent and we have

$$
\begin{gathered}
\frac{k}{2(k+x)}\left[\prod_{n=1}^{\infty}\left(1-\frac{1}{4 n^{2}}\right)\right]^{1 / 4}\left[\prod_{n=1}^{\infty}\left(1-\frac{1}{16 n^{2}}\right)\right]^{1 / 2} \\
\cdot\left[\prod_{n=1}^{\infty}\left(1-\frac{1}{(2 n+1)^{2}}\right)\right]^{-1 / 4} \cdot
\end{gathered}
$$

Now it is possible to give the numerical value of these product terms since

$$
\begin{aligned}
& \prod_{n=1}^{\infty}\left(1-1 / 4 n^{2}\right)=\left.\frac{\sin x}{x}\right|_{x=\pi / 2}=\frac{2}{\pi}, \\
& \prod_{n=1}^{\infty}\left(1-1 / 16 n^{2}\right)=\left.\frac{\sin x}{x}\right|_{x=\pi / 4}=\frac{4}{\pi \sqrt{2}}
\end{aligned}
$$

and

$$
\prod_{n=1}^{\infty}\left(1-1 /(2 n+1)^{2}\right)=\lim _{x \rightarrow \pi / 2} \frac{\cos x}{1-4 x^{2} / \pi^{2}}=\frac{\pi}{4} \text {. }
$$


Hence (6.5) reduces to (6.4). The advantage of this latter calculation is that it permits us to evaluate the phase angle of the transmission amplitude if it is required.

\section{Appendix}

\section{A The Additive Decomposition of $\ln D(w)$ and $\ln E(w) / \sigma$}

In this section we will give the explicit decomposition of $\ln D(w)$ and $\ln E(w) / \sigma$ into additive components which are analytic in the appropriate upper and lower half planes. We note to start that the treatment of $\ln D(w)$ is particularly simple since most of the required information can be found in certain scalar problems [1]. Two cases may occur when we only ask for specular reflection in free space. In the first place, only one mode will propagate in a duct if $\pi / 2<a k<3 \pi / 2$ and this implies in the present instance that $\varkappa^{2}=k^{2}$ $-\pi^{2} / 4 a^{2}>0$. On the other hand we will have specular reflection if $0<a k$ $<2 \pi$. Hence for the simultaneous propagation of a single mode in a duct and specular reflection in free space we require $\pi / 2<a k<3 \pi / 2$.

For this case

$$
\begin{aligned}
\ln D(w) & =\frac{1}{2} \ln \left(-\frac{\cos a \sigma}{\sin ^{2} a \sigma / 2}\right) \\
& =\frac{1}{2} \ln \frac{16}{\pi^{2}} \frac{\left(k^{2}-w^{2} \prod_{n=1}^{\infty}\left[1-\frac{4 a^{2}\left(k^{2}-w^{2}\right)}{(2 n+1)^{2} \pi^{2}}\right]\right.}{\left(k^{2}-w^{2}\right)}\left\{\prod_{n=1}^{\infty}\left[1-\frac{a^{2}\left(k^{2}-w^{2}\right)}{4 n^{2} \pi^{2}}\right]\right\}^{2}
\end{aligned}
$$

Observe that we have chosen the principal branch of the logarithm and since $\pi / 2<a k<3 \pi / 2,-\cos a \sigma$ is real and positive for $w=0$. Hence

$$
\begin{aligned}
& \ln D_{+}(w)=\frac{1}{2}\left\{\sum_{n=1}^{\infty}\left(\ln \left[\sqrt{1-\frac{4 a^{2} k^{2}}{\pi^{2}(2 n+1)^{2}}}-\frac{2 \mathrm{i} a w}{(2 n+1) \pi}\right]+\frac{2 \mathrm{i} a w}{(2 n+1) \pi}\right)\right. \\
& \text { (A.1) } \left.\quad-2 \sum_{n=1}^{\infty}\left(\ln \left[\sqrt{1-\frac{a^{2} k^{2}}{4 n^{2} \pi^{2}}}-\frac{\mathrm{i} a w}{2 n \pi}\right]+\frac{\mathrm{i} a w}{2 n \pi}\right)+\chi(w)\right\}
\end{aligned}
$$

where $\ln D_{+}(w)$ is analytic in the upper half-plane $\operatorname{Im}>-\sqrt{\frac{9 \pi^{2}}{4 a^{2}}-k^{2}}$. The factor $\chi(w)$ will be chosen to give $\ln D_{+}(w)$ algebraic behavior for $|w| \rightarrow \infty$ in this half-plane. The linear factors following the logarithmic terms on the right side of (A.1) have been inserted to make the infinite sums absolutely convergent. Similarly 
(A.2) $\ln D_{-}(w)=\frac{1}{2} \ln \frac{16}{\pi^{2}} \frac{\left(\kappa^{2}-w^{2}\right)}{\left(k^{2}-w^{2}\right)}+\ln D_{+}(-w)$

is analytic in the lower half-plane $\operatorname{Im} w<0$.

We now turn to the determination of the function $\chi(w)$. Recall that for $\operatorname{Im} w>-\sqrt{\frac{9 \pi^{2}}{4 a^{2}}-k^{2}}$ and $|w| \rightarrow \infty, \ln D_{+}(w)$ is independent of $a k$ [7]. Hence the dominant behavior of $D_{+}(w)$ in this half-plane at infinity can be derived from

(A.3)

$$
\frac{\left\{\exp [\chi(w)] \prod_{n=1}^{\infty}\left[1-\frac{2 \mathrm{i} a w}{(2 n+1) \pi}\right] \exp (2 \mathrm{i} a w /(2 n+1) \pi)\right\}}{\left\{\prod_{n=1}^{\infty}\left[1-\frac{\mathrm{i} a w}{2 n \pi}\right] \exp (\mathrm{i} a w / 2 n \pi)\right\}^{2}}
$$

Now (A.3) can be rewritten as

(A.4)

$$
\frac{\left\{\exp [\chi(w)] \prod_{n=2}^{\infty}\left[1-\frac{2 \mathrm{i} a w}{n \pi}\right] \exp (2 \mathrm{i} a w / n \pi)\right\}}{\prod_{n=1}^{\infty}\left[1-\frac{\mathrm{i} a w}{n \pi}\right] \exp (\mathrm{i} a w / n \pi)\left\{\prod_{n=1}^{\infty}\left[1-\frac{\mathrm{i} a w}{2 n \pi}\right] \exp (\mathrm{i} a w / 2 n \pi)\right\}^{2}},
$$

(A.4) can be expressed in terms of the gamma function as

$$
\frac{-a^{2} w^{2} \Gamma^{2}(-\mathrm{i} a w / 2 \pi) \Gamma(-\mathrm{i} a w / \pi)}{8 \pi(\pi-2 \mathrm{i} a w) \Gamma(-2 \mathrm{i} a w / \pi)} \exp [\chi(w)-2 \mathrm{i} a w / \pi] .
$$

With the aid of the Stirling expansion we find that when $|w| \rightarrow \infty, \operatorname{Im} w>$ $-\sqrt{\frac{9 \pi^{2}}{4 a^{2}}-k^{2}}$ that the dominant term in (A.3) is

$$
(\pi \sqrt{2} / 4) \exp [\chi(w)+(3 \mathrm{i} a w \ln 2-2 \mathrm{i} a w) / \pi] .
$$

In particular when $\chi(w)$ is chosen ia $w(2-3 \ln 2) / \pi, \ln D_{+}(w)$ is bounded in this half-plane. The same factor $\chi(w)$ makes $D_{-}(w)$ bounded in lower half-plane $\operatorname{Im} w<0,|w| \rightarrow \infty$.

We now turn to a somewhat more subtle task, namely the additive decomposition of

$$
\text { (A.5) } \frac{1}{2 \sigma} \ln \frac{\sin \left(\frac{a \sigma}{2}+\frac{\pi}{4}\right)}{\sin \left(\frac{a \sigma}{2}-\frac{\pi}{4}\right)}
$$


where we have chosen the principal branch of the logarithm in (A.5) for $w=0$ when $\pi / 2<a k<3 \pi / 2$. We write a product representation for the ratio of the trigonometric functions in order to reveal the singularities of the logarithmic function in (A.5) to obtain

$$
\frac{\sin \left(\frac{a \sigma}{2}+\frac{\pi}{4}\right)}{\sin \left(\frac{a \sigma}{2}-\frac{\pi}{4}\right)}=\frac{(2 a \sigma+\pi)}{(2 a \sigma-\pi)} \prod_{n=1}^{\infty} \frac{\left[1-\left(\frac{\pi+2 a \sigma}{4 n \pi}\right)^{2}\right]}{\left[1-\left(\frac{2 a \sigma-\pi}{4 n \pi}\right)^{2}\right]}
$$

$$
=\frac{(2 a \sigma+\pi)^{2}}{4 a^{2}\left(x^{2}-w^{2}\right)}\left\{\prod_{n=1}^{\infty} \frac{[1+(\pi+2 a \sigma) / 4 n \pi]}{[1+(2 a \sigma-\pi) / 4 n \pi]} \exp (-1 / 2 n)\right\}^{2} \quad L(w) L(-w)
$$

where $L(w)=\prod_{n=1}^{\infty}\left\{\frac{\sqrt{\left(1-\frac{1}{4 n}\right)^{2}-\frac{a^{2} k^{2}}{4 n^{2} \pi^{2}}}+\frac{\mathrm{i} a w}{2 n \pi}}{\sqrt{\left(1+\frac{1}{4 n}\right)^{2}-\frac{a^{2} k^{2}}{4 n^{2} \pi^{2}}}+\frac{\mathrm{i} a w}{2 n \pi}}\right\} \exp (1 / 2 n)$.

The exponential factors have been inserted in the above infinite products in order to make them absolutely convergent in the strip of analyticity $-\sqrt{\frac{9 \pi^{2}}{4 a^{2}}-k^{2}}$ $<\operatorname{Im} w<0$ and permit us to form the above preliminary decomposition. The

$$
\text { (A.7) } \frac{1}{2 \sigma} \ln \frac{(2 a \sigma+\pi)^{2}}{4 a^{2}\left(x^{2}-w^{2}\right)}\left\{\prod_{n=1}^{\infty} \frac{\left[1+\left(\frac{\pi+2 a \sigma}{4 n \pi}\right)\right]}{\left[1+\left(\frac{2 a \sigma-\pi}{4 n \pi}\right)\right]} \exp (-1 / 2 n)\right\}^{2} L(w)
$$

is analytic in the lower half-plane $\operatorname{Im} w<0$ while the term $[\ln L(-w)] / 2 \sigma$ is only analytic in the strip $-\sqrt{\frac{9 \pi^{2}}{4 a^{2}}-k^{2}}<\operatorname{Im} w<0$.

However $[\ln L(-w)] / 2 \sigma$ may be decomposed additively with the aid of the Cauchy integral theorem. We form the integral

(A.8) $\frac{1}{2 \pi \mathrm{i}} \int_{P} \frac{\ln L(-t) \mathrm{d} t}{\sigma(t)(t-w)}$

where $P$ is a closed path taken over the rectangle whose horizontal sides are $\alpha-\mathrm{i} \varepsilon_{1}$ and $\alpha-\mathrm{i} \varepsilon_{2},-K<\alpha<K$ and

$$
-\sqrt{\frac{9 \pi^{2}}{4 a^{2}}-k^{2}}<-\varepsilon_{1}<-\varepsilon_{2}<0 \text {. }
$$


The vertical sides of the rectangle are $-K+\mathrm{i} \beta$ and $K+\mathrm{i} \beta,-\varepsilon_{1}<\beta<-\varepsilon_{2}$. With $w$ interior to this rectangle, it is a simple task to see that there are no contributions to (A.8) from the vertical sides of $P$ when $K \rightarrow \infty$. Hence

$$
2 \pi \mathrm{i} \frac{\ln L(-w)}{\sigma(w)}=\int_{-\mathrm{i} \varepsilon_{1}-\infty}^{-\mathrm{i} \varepsilon_{1}+\infty} \frac{\ln L(-t) \mathrm{d} t}{\sigma(t)(t-w)}-\int_{-\mathrm{i} \varepsilon_{2}-\infty}^{-\mathrm{i} \varepsilon_{2}+\infty} \frac{\ln L(-t) \mathrm{d} t}{\sigma(t)(t-w)}
$$

where the first integral on the right side is analytic in the upper half-plane $\operatorname{Im} w$ $>-\varepsilon_{1}$ while the second one is analytic in the lower half-plane $\operatorname{Im} w<-\varepsilon_{2}$. Since the infinite series in $\ln L(-w)$ being absolutely convergent, it may be integrated termwise in a sense which we shall presently describe. It will also be shown that the portion of $[\ln L(-w)] / \sigma(w)$ which is analytic in the upper halfplane $\operatorname{Im} w>-\sqrt{\frac{9 \pi^{2}}{4 a^{2}}-k^{2}}$ can be reduced to

(A.9) $\mathrm{i} \sum_{n=1}^{\infty} \int_{\beta_{n}}^{\infty} \frac{\mathrm{d} \tau}{\sqrt{k^{2}+\tau^{2}}(\mathrm{i} \tau+w)}-\mathrm{i} \sum_{n=1}^{\infty} \int_{\beta}^{\infty} \frac{\mathrm{d} \tau}{\sqrt{k^{2}+\tau^{2}}(\mathrm{i} \tau+w)}$.

Upon evaluating the integrals in (A.9) we finally obtain

$$
-\frac{1}{\sigma(w)} \sum_{n=1}^{\infty} \ln \frac{w+\mathrm{i} \beta_{n}}{w+\mathrm{i} \beta_{-n}} M(w)
$$

where $\beta_{n}=\frac{2|n| \pi}{a} \sqrt{\left(1+\frac{1}{4 n}\right)^{2}-\frac{a^{2} k^{2}}{4 n^{2} \pi^{2}}}$

and $\quad M(w)=\frac{\sqrt{\left(\beta_{-n}^{2}+k^{2}\right)\left(k^{2}-w^{2}\right)}+\mathrm{i} w \beta_{-n}+k^{2}}{\sqrt{\left(\beta_{n}^{2}+k^{2}\right)\left(k^{2}-w^{2}\right)}+\mathrm{i} w \beta_{n}+k^{2}}$.

This sum can be rewritten as

$\frac{2 \ln H_{1}(w)}{\sigma(w)}=-\frac{1}{\sigma(w)} \ln \prod_{n=1}^{\infty}\left[\frac{\sqrt{\left(1+\frac{1}{4 n}\right)^{2}-\frac{a^{2} k^{2}}{4 n^{2} \pi^{2}}}-\frac{\mathrm{i} a w}{2 n \pi}}{\sqrt{\left(1-\frac{1}{4 n}\right)^{2}-\frac{a^{2} k^{2}}{4 n^{2} \pi^{2}}}-\frac{\mathrm{i} a w}{2 n \pi}}\right] \exp (-1 / 2 n)$

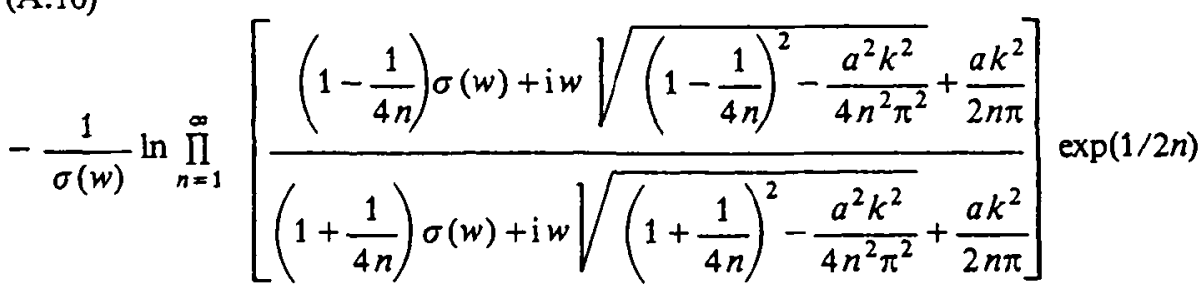


In order to derive (A.9) we observe that for a given $n$, we are concerned with the evaluation of a term of the form

$$
\text { (A.11) } \frac{1}{2 \pi \mathrm{i}} \int_{-\mathrm{i} \varepsilon_{1}-\infty}^{-\mathrm{i} \varepsilon_{1}+\infty}\left[\ln \frac{\left(t+\mathrm{i} \beta_{-n}\right)}{\left(t+\mathrm{i} \beta_{n}\right)}+\frac{1}{2 n}\right] \frac{\mathrm{d} t}{\sigma(t)(t-w)} \text {. }
$$

The second integral vanishes. The first integral may be evaluated by noting that the integrand has branch points at $t=-\mathrm{i} \beta_{n}$ and $-\mathrm{i} \beta_{-n}$. We draw branch cuts from $-\mathrm{i} \beta_{n}$ to $-\mathrm{i} \infty$ and from $-\mathrm{i} \beta_{-n}$ to $-\mathrm{i} \infty$. Then $\ln \left(t+\mathrm{i} \beta_{n}\right)=\ln \left|t+\mathrm{i} \beta_{n}\right|$ $-\mathrm{i} \pi / 2$ on the right side of the cut from $-\mathrm{i} \beta_{n}$ and it is equal to $\ln \left|t+\mathrm{i} \beta_{n}\right|+3 \mathrm{i} \pi / 2$ on the left side of the same cut. The path from $-\mathrm{i} \varepsilon_{1}-R$ to $-\mathrm{i} \varepsilon_{1}+R$ may be deformed into the half-plane to circular arcs of radius $R$ in the third and fourth quadrants. These arcs are joined by a "hairpin" path which follows the left and right sides of the cut. When $R \rightarrow \infty$, the contributions from the circular arcs vanish and (A.11) reduces to the corresponding terms in (A.9).

Rather than evaluating the integral

$$
-\frac{1}{2 \pi \mathrm{i}} \int_{-\mathrm{i} \varepsilon_{2}-\infty}^{-\mathrm{i} \varepsilon_{2}+\infty} \frac{\ln L(-t) \mathrm{d} t}{\sigma(t)(t-w)}
$$

in order to complete the additive decomposition of $L(-w)$, we shall simply form

$$
\frac{\ln L(-w)}{\sigma(w)}-\frac{1}{2 \pi \mathrm{i}} \int_{-\mathrm{i} \varepsilon_{1}-\infty}^{-\mathrm{i} \varepsilon_{1}+\infty} \frac{\ln L(-t) \mathrm{d} t}{\sigma(t)(t-w)}
$$

and obtain for (A.12)

$$
\frac{\ln M(w)}{\sigma(w)} \text {. }
$$

This last expression is clearly analytic in the lower half-plane $\operatorname{Im} w<0$. With it we can now complete the additive decomposition of (A.5). Upon bringing together the various terms which we have found in this Appendix, we have that

$$
\begin{aligned}
& \frac{1}{2 \sigma} \ln \frac{(2 a \sigma+\pi)^{2}}{4 a^{2}\left(\varkappa^{2}-w^{2}\right)}\left\{\prod_{n=1}^{\infty}\left[\frac{1+\left(\frac{\pi+2 a \sigma}{4 n \pi}\right)}{1+\left(\frac{2 a \sigma-\pi}{4 n \pi}\right)}\right] \exp (-1 / 2 n)\right\}^{2} \\
& \cdot L(w) M(w)=\frac{\ln M_{2}(w)}{\sigma}
\end{aligned}
$$

is that component of (A.5) (in the additive sense) which is analytic in the lower half-plane $\operatorname{Im} w<0$.

\section{B Verification of the Single-Valuedness of (A.10)}

It is a simple task to verify that (A.10) is analytic in the upper half-plane $\operatorname{Im} w>-\sqrt{\frac{9 \pi^{2}}{4 a^{2}}-k^{2}}$. We note first that $\ln \left(w+\mathrm{i} \beta_{-n}\right) /\left(w+\mathrm{i} \beta_{n}\right)$ is analytic 
in this half plane since it only has branch points at $w=-\mathrm{i} \beta_{n}, n= \pm 1, \pm 2, \ldots$ In particular the branch point with the smallest magnitude is $-\mathrm{i} \beta_{-1}$. The factor $\sigma(w)=\sqrt{k^{2}-w^{2}}$ which we have taken to be $k$ when $w=0$ is analytic in the cut $w$ plane, cut from $-\infty$ to $-k$ and from $k$ to $\infty$. If we put $w=u+\mathrm{i} v, v \rightarrow 0^{-}$, $u>k,(\mathrm{~A} .10)$ becomes

$$
\begin{aligned}
& -\frac{1}{\mathrm{i} \sqrt{u^{2}-k^{2}}}\left\{\sum_{n=1}^{\infty} \ln \left[\frac{\mathrm{i} \sqrt{\left(k^{2}+\beta_{n}^{2}\right)\left(u^{2}-k^{2}\right)}+\mathrm{i} u \beta_{n}+k^{2}}{u+\mathrm{i} \beta_{n}}\right]\right. \\
& \left.-\sum_{n=1}^{\infty} \ln \left[\frac{\mathrm{i} \sqrt{\left(k^{2}+\beta_{-n}^{2}\right)\left(u^{2}-k^{2}\right)}+\mathrm{i} u \beta_{-n}+k^{2}}{u+\mathrm{i} \beta_{-n}}\right]\right\} \\
& =\frac{1}{\mathrm{i} \sqrt{u^{2}-k^{2}}}\left\{\sum_{n=1}^{\infty} \ln \left[\frac{-\mathrm{i} \sqrt{\left(k^{2}+\beta_{n}^{2}\right)\left(u^{2}-k^{2}\right)}+\mathrm{i} u \beta_{n}+k^{2}}{u+\mathrm{i} \beta_{n}}\right]\right. \\
& \left.-\sum_{n=1}^{\infty} \ln \left[\frac{-\mathrm{i} \sqrt{\left(k^{2}+\beta_{-n}^{2}\right)\left(u^{2}-k^{2}\right)}+\mathrm{i} u \beta_{-n}+k^{2}}{u+\mathrm{i} \beta_{-n}}\right]\right\}
\end{aligned}
$$

after some simplification. But the right side of this equality is Lim (A.10), $v \rightarrow 0+, u>k$. There is a similar remark to be made when $v \rightarrow 0$ - or $0+$, $u<-k$. Hence (A.10) is analytic and single valued in the upper half-plane $\operatorname{Im} w>-\sqrt{\frac{9 \pi^{2}}{4 a^{2}}-k^{2}}$.

\section{Added in proof}

After the present paper was accepted and was already in the press, we were apprised of a paper by E. Lüneburg and R. A. Hurd "Diffraction by an infinite set of soft/ hard parallel half planes" which appeared in Radio Science 17 (1982) 453-462 and which dealt with the same problem. Our formulation as well as our use of the methods of complex analysis is entirely different from theirs and we believe they are of independent interest.

\section{References}

[1] Carlson, J. F.; Heins, A. E.: The Reflection of an Electromagnetic Plane Wave by an Infinite Set of Plates I. Quart. Appl. Math. 4 (1947) $313-329$

Heins, A. E.; Carlson, J. F.: The Reflection of an Electromagnetic Plane Wave by an Infinite Set of Plates II. Quart. Appl. Math. 5 (1947) 82-88

[2] Heins, A. E.: The Green's Function for Periodic Structures in Diffraction Theory with an Application to Parallel Plate Media, I and II. J. Math. Mech. 6 (1957) 401-426; 629-639

[3] Heins, A. E.: System of Wiener-Hopf Integral Equations and Their Application to Some Boundary Value Problems in Electromagnetic Theory. Proc. Symp. Appl. Math., Am. Math. Soc., Vol. 2 (1950) 76-81

Heins, A. E.: The Sommerfeld Half-Plane Problem Revisited, II; The Factoring of a Matrix of Analytic Functions. Math. Meth. in the Appl. Sci. 5 (1983) 14-21

[4] Korevaa, J.: Mathematical Methods, Vol I. New York-London: Academic Press 1968 
[5] Laneburg, E.: Diffraction by an Infinite Set of Parallel Half Planes. Kleinheubacher Berichte (ed. by FTZ Darmstadt, W. Germany) 24 (1981) 17-35

[6] Rawlins, A. D.: The Solution of a Mixed Boundary Value Problem in the Theory of Diffraction by a Semi-Infinite Plane. Proc. R. Soc. Lond. A 346 (1975) $469-484$

[7] Schwinger, J. S.; Saxon, D. S.: Discontinuities in Wave Guides (Notes on Lectures by Julian Schwinger). New York-London-Paris: Gordon and Breach 1968

Professor Albert E. Heins

Department of Mathematics

University of Michigan

Ann Arbor, Michigan 48109, U.S.A.

(Received January 22, 1982) 